\title{
FRÉQUENCE ET IMPORTANCE DU CHARRIAGE DANS LES RIVIĖRES DU MASSIF ARDENNAIS
}

Geoffrey HOUBRECHTS, Éric HALLOT, Frédéric GOB, Julien MOLS, Olivier DEFECHEREUX et François PETIT*; Laboratoire d'hydrographie et de géomorphologie fluviatile, Université de Liège, Allée du 6 Août, 2 (Bâtiment B11-Sart Tilman), B-4000 Liège, Belgique.

RÉSUMÉ L'analyse du transport de la charge de fond de plusieurs rivières du massif ardennais (Belgique) par différentes techniques de marquage de galets (peinture, charge métallique, émetteurs radio) a permis d'identifier le débit critique où débute le charriage. Pour les rivières plus importantes (plus de $500 \mathrm{~km}^{2}$ ), la mise en mouvement se produit à des débits légèrement inférieurs au débit à plein bord $(0,7$ à $1 Q_{b}$ ), débits qui se présentent en moyenne 5 à 11 jours par an. Pour les rivières de taille intermédiaire (entre 100 et $500 \mathrm{~km}^{2}$ ), le charriage débute à un débit avoisinant 0,5 fois le débit à plein bord, avec une récurrence de 0,3 an et une durée variant entre 8 et 12 jours par an. Pour les rivières dimension modeste (moins de $100 \mathrm{~km}^{2}$ ), la mise en mouvement se produit à des débits compris entre 0,5 et $0,8 Q_{b}$, mais leur récurrence est relativement faible et le charriage peut se produire jusqu'à 20 jours par an. Par ailleurs, le transport solide a été évalué à l'aide de pièges à sédiments dans les rivières de petite dimension (moins de $10 \mathrm{~km}^{2}$ ); il est relativement peu important dans les bassins forestiers $\left(0,5 \mathrm{t} / \mathrm{km}^{2} / \mathrm{an}\right)$, en raison de la multiplication des embâcles végétaux qui accroissent la rugosité du lit. Pour les rivières plus importantes, le transport solide a été estimé entre 0,4 et $2,5 \mathrm{t} / \mathrm{km}^{2} / a n$ grâce à l'analyse des quantités curées systématiques aux mêmes sites et à la réalisation de levés topographiques de contrôle. Nous avons ensuite analysé l'évolution des quantités charriées en fonction de la puissance spécifique à plein bord développée par ces rivières. La relation établie sur la base de ces données a permis de mettre en évidence le déficit en sédiments de certaines rivières ainsi que plusieurs facteurs influençant le charriage.
ABSTRACT Frequency and extent of bedload transport in rivers of the Ardenne. The analysis of bedload transport of several rivers of the Ardenne (Belgium) using various marking techniques (painted pebbles metallic-tagged pebbles, radio-transmitters) has allowed to identify the critical discharge when bedload transport begins. In large rivers (more than $500 \mathrm{~km}^{2}$ ), bedload mobilisation occurs when the discharge is slightly lower than the bankfull $\left(0.7\right.$ to $\left.1 Q_{b}\right)$, which takes place on average 5 to 11 days per year. In medium size rivers (between 100 and $500 \mathrm{~km}^{2}$ ), bedload transport occurs when the discharge is around $0.5 \mathrm{Q}_{\mathrm{b}}$, which corresponds to a recurrence of approximately 0.3 years and to a mobilisation period of 8 to 12 days per year. In smaller rivers (less than $100 \mathrm{~km}^{2}$ ), the critical discharge occurs between 0.5 and $0.8 Q_{b}$, but its reccurence is relatively low and bedload can be mobilized up to 20 days per year. Moreover, the bedload solid discharge was estimated using sediment traps in headwater streams (less than $10 \mathrm{~km}^{2}$ ). In small forested catchments, bedload transport is not significant (around $0.5 \mathrm{t} / \mathrm{km}^{2} / \mathrm{yr}$ ), partially due to numerous vegetation log-jams increasing bed roughness. In larger rivers, the specific bedload discharge was estimated between 0.4 and $2.5 \mathrm{t} / \mathrm{km}^{2} / \mathrm{yr}$ using quantities of dredged material extracted systematically in the same place, in relation to topographical surveys. Finally, the evolution of the bedload discharge in relation to the specific stream power at the bankfull discharge was analysed. The relation obtained with this data shows the deficit of sediment in some rivers and explains some of the factors that influence bedload transport. 


\section{INTRODUCTION}

Les études récentes sur le transport de la charge de fond sont relativement nombreuses. Elles portent notamment sur les différentes techniques de marquage (Ergenzinger et al., 1989; Hassan et Ergenzinger, 2003; Lamarre et al., 2005), sur la tendance qu'a la charge de fond à se mouvoir sous forme de vagues (Gomez et al., 1989; Hassan et al., 1992; Hoey, 1992; Ferguson et al., 2002), sur les critères d'entraînement basés sur les débits unitaires, les tensions de cisaillement et les puissances spécifiques (Bathurst et al., 1979; Ferguson, 1994 ; Martin, 2003; Petit et al., 2005), sur l'influence des effets de saillie et de masquage (Reid et Frostick, 1984 ; Richards, 1988, 1990), et sur les variations des tensions de cisaillement au sein même du lit qui peuvent influencer localement les quantités charriées (Powell et Ashworth, 1995).

Toutefois, peu d'études nous renseignent sur l'occurrence du débit requis pour mobiliser la charge de fond, sur la fréquence du charriage et sur l'estimation des quantités charriées. Il est globalement admis que le charriage dans des rivières à charge de fond caillouteuse nécessite des débits relativement importants et que, de ce fait, il ne se réalise que de façon épisodique (Leopold et al., 1964). Longtemps, on a considéré que la mobilisation et le transport de la charge caillouteuse ne se produisaient que pour des débits proches du débit à plein bord $\left(Q_{b}\right)$, c'est-à-dire en moyenne une fois par an pour des rivières importantes (Tricart, 1965, 1977). Toutefois, par la suite, plusieurs études ont abouti à nuancer ce propos et à avancer des débits de mobilisation nettement plus fréquents, généralement inférieurs à $0,5 \mathrm{Q}_{\mathrm{b}}$ (tabl. I). Ainsi, Thorne et Lewin (1979) ont montré que la mobilisation débutait pour un débit égal à $0,24 Q_{b}$, qui s'est présenté 25 fois en deux ans. Dans le Rhône, Tricart et Vogt (1967) ont déterminé que le charriage des galets de petite taille se produit de 20 à 40 jours par an (soit 5 à $10 \%$ du temps), ce qui ressort également des expériences menées plus récemment (Petit et al., 1996). Dans la Laimbach, torrent bavarois caractérisé par une organisation en step-pool, le débit de mobilisation représente moins du cinquième du débit dont la récurrence est 1,5 an et se présente en moyenne 3 fois par an (Gintz et al., 1996). Par ailleurs, le début de mobilisation dans une rivière du Colorado représente environ $0,4 \mathrm{Q}_{\mathrm{b}}$ alors que, dans l'East Fork River (Wyoming), la mobilisation débute pour un débit moindre que le débit moyen annuel $\left(0,15 Q_{b}\right)$ (Leopold ,1994).

Par ailleurs, contrairement à la charge transportée en suspension, l'évaluation des quantités transportées par charriage présente beaucoup plus de difficultés techniques et méthodologiques (Reid et al., 1985; Miyamoto et al., 1992), ce qui explique que les études portant sur ce sujet sont relativement peu nombreuses. D'une manière générale, on s'accorde à reconnaître que les quantités transportées par charriage ne représentent qu'une faible partie des quantités de sédiments exportés hors des bassins. Ainsi, Reid et al. (1998) considèrent qu'en milieu océanique, le rapport entre les quantités charriées et les quantités transportées en suspension est inférieur à $10 \%$, mais ce ratio augmente nettement en milieu montagnard. Toutefois, il se dégage une grande variabilité des quantités transportées, en fonction du système morphoclimatique, des caractéristiques intrinsèques des rivières considérées (pente, débit, taille des sédiments, présence d'embâcles), de leur héritage géomorphologique et de la disponibilité en matériaux. Ainsi, les quantités peuvent varier de $1 \mathrm{t} / \mathrm{km}^{2} /$ an pour de petits bassins forestiers sous climat tempéré océanique (Newson, 1980) à plus de 100, voire $300 \mathrm{t} / \mathrm{km}^{2} / \mathrm{an}$ dans des torrents alpins (Vezzoli, 2004) ou des rivières méditerranéennes à forte énergie du relief (Liébault, 2003; Piégay et al., 2004).

Des estimations de transport solide avaient déjà été avancées précédemment pour une dizaine de rivières du massif ardennais, avec des valeurs variant de 0,4 à 2,2 $\mathrm{t} / \mathrm{km}^{2} / \mathrm{an}$ (Petit et al., 1996). Depuis lors, de nombreuses campagnes de marquage ont été effectuées sur d'autres rivières, de différentes tailles et situées dans des contextes géomorphologiques régionaux diversifiés. Ces observations supplémentaires ont permis d'analyser de manière plus précise la dynamique de la charge de fond pour une gamme de rivières variées. Par ailleurs, des traitements statistiques ont été appliqués à des séries de débits plus longues, en faisant intervenir des ajus-

TABLEAU I

Exemples de valeurs de débit de mise en mouvement (exprimé par rapport au débit à plein bord) et fréquence du charriage

\begin{tabular}{|c|c|c|c|c|}
\hline & $\begin{array}{l}\text { Débit de mise en } \\
\text { mouvement exprimé } \\
\text { par rapport au débit } \\
\text { à plein bord }\end{array}$ & $\begin{array}{l}\text { Nombre de jours de } \\
\text { charriage exprimé } \\
\text { en \% de l'année }\end{array}$ & $\begin{array}{l}\text { Taille du matériau } \\
\text { composant le lit } \\
(\mathrm{mm})\end{array}$ & Référence \\
\hline Severn & 0,24 & 25 fois en 2 ans & 28 & Thorne et Lewin, 1979 \\
\hline Colorado Mountain & 0,4 & $15 \%$ du temps & & Leopold, 1994 \\
\hline Wyoming & 0,15 & $20 \%$ du temps & & Leopold, 1994 \\
\hline Rhône Lyon & & 5 à $10 \%$ du temps & Galets & Tricart, 1967 \\
\hline Rhône Lyon (Miribel) & $0,3-0,44$ & 8 à $11 \%$ du temps & 23,2 & Petit et al., 1996 \\
\hline Laimbach (Bavière) & 0,14 (a) & 3 fois par an & $50-800^{(b)}$ & Gintz et al., 1996 \\
\hline Sagehen Creek (Californie) & 0,5 & $3,3 \%$ du temps & $58\left(26^{(\mathrm{c})}\right)$ & Andrews, 1994 \\
\hline
\end{tabular}

(a) Par rapport au débit de récurrence 1,5 an il s'agit d'un torrent en step-pool.

(b) Mobilisation d'éléments marqués plus petits que le matériau constituant les steps.

(c) Taille des sédiments charriés. 
tements plus fins, ce qui a permis de préciser la récurrence des débits de mobilisation de la charge de fond. L'analyse des hydrogrammes des différentes rivières a également permis d'estimer le nombre moyen de jours par an durant lequel le charriage se produit. Enfin, pour estimer le débit solide de la charge de fond, nous avons eu accès à une série d'archives complémentaires fournies par les gestionnaires des cours d'eau, ce qui a permis d'élargir notre champ d'investigation en nombre de rivières étudiées et d'augmenter le nombre d'observations par rivière.

\section{CARACTÉRISTIQUES DES RIVIĖRES ÉTUDIÉES}

La plupart des rivières étudiées prennent leur source en Ardenne et s'écoulent en direction de la Meuse en traversant différentes régions naturelles (fig. 1). Les plus petites d'entre elles possèdent un bassin versant inférieur à $1 \mathrm{~km}^{2}$, le plus grand atteint $3000 \mathrm{~km}^{2}$. Toutes ces rivières ont une pente relativement forte, variant de plus de 50 pour mille en tête de bassin à moins de 1 pour mille pour les plus importantes. Les puissances spécifiques (pour le débit à plein bord) sont comprises entre 25 et $150 \mathrm{~W} / \mathrm{m}^{2}$ (Petit et al., 2005). Ce sont des rivières à chenal unique où la méandration est relativement bien développée au sein d'une plaine alluviale constituée d'une nappe caillouteuse héritée de la dernière période glaciaire (Juvigné, 1979). Cette nappe repose sur la roche en place, rarement atteint par les rivières (Houbrechts, 2005), et est elle-même surmontée par une couche limoneuse de 1 à 2 m d'épaisseur (Petit, 1995).

Les rivières sélectionnées possèdent une charge de fond caillouteuse et leur bassin versant est constitué de roches imperméables. Un premier ensemble comprend des rivières situées au sud de l'Ardenne avec la Rulles (1) et la Semois ardennaise (2). II s'agit de rivières à pente faible et développant une puissance spécifique au $Q_{b}$ inférieure à $30 \mathrm{~W} / \mathrm{m}^{2}$. Le rapport largeur/profondeur y est relativement important et atteint par endroit 30 (Gob et al., 2005).

Un second ensemble regroupe les rivières descendant de la retombée septentrionale de l'Ardenne. II s'agit de l'Eau Noire (3), la Lesse (4-6), la Lhomme (7 et 8), la Wamme (9), l'Ourthe (10-14), le Ruisseau de Belleva (15), le Ruisseau de la Mer (16), l'Aisne (17 et 18), la Chavanne (19), la Lienne (20), l'Amblève (21), le Ru des Waidages (22), la Hoëgne (23) et la Vesdre (24). Ce sont des rivières à pente forte avec des

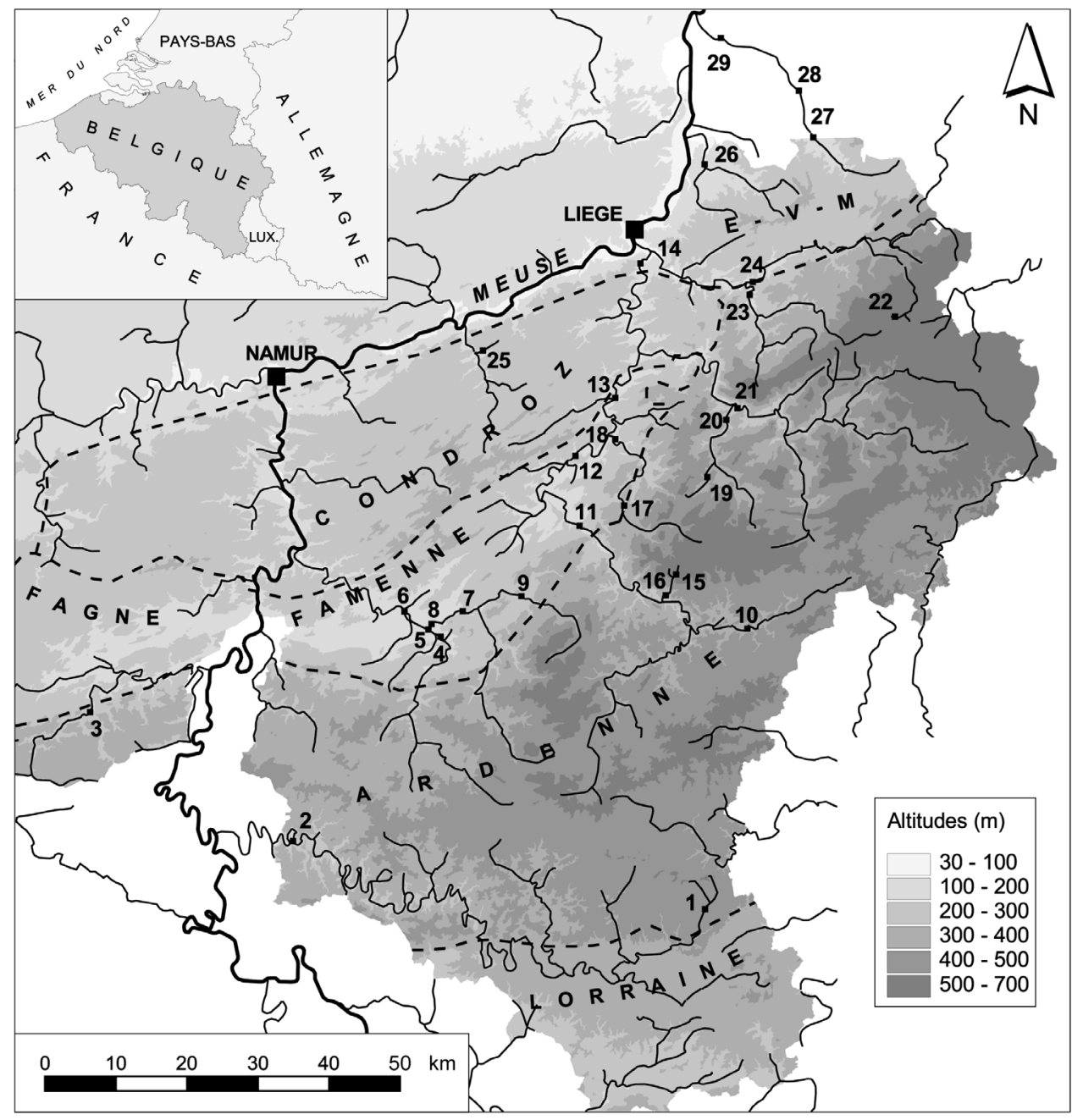

FIGURE 1. Carte de localisation des sites de mesures: la numérotation renvoie aux tableaux I et III. Location map of the studied sites: numbers refer to tables I and III. 
puissances au $Q_{b}$ généralement comprises entre 50 et $100 \mathrm{~W} / \mathrm{m}^{2}$, pouvant atteindre $180 \mathrm{~W} / \mathrm{m}^{2}$ (Petit et al., 2005). La plupart de ces rivières possèdent une charge de fond constituée d'éléments caillouteux $\left(D_{50}\right.$ des seuils compris entre 5 et $10 \mathrm{~cm}$ ) (Houbrechts, 2005).

Un troisième ensemble de rivières a également été retenu. II regroupe des rivières à charge caillouteuse développées partiellement sur substrat perméable. Elles sont situées soit dans le Condroz (Ruisseau de Wavelinse, 25), soit dans l'Entre-Vesdre-et-Meuse (EVM sur la fig. 1) (Berwinne, 26; Gueule, 27-29).

Précisons que certaines rivières traversent plusieurs régions naturelles mais qu'elles conservent leur caractère ardennais sur l'ensemble de leur cours, en ce qui concerne leur charge caillouteuse et le régime des débits. C'est par exemple le cas de la Lesse (5 et 6), de la Lhomme (8) et de l'Ourthe (11-14) que nous considérerons comme des rivières ardennaises bien que les stations de mesure de débits et les sites d'étude se situent en Fagne-Famenne ou dans le Condroz.

Le régime des débits des rivières ardennaises est contrasté, car elles sont développées sur un substrat imperméable (phyllades, quartzophyllades et quartzites du Cambrien et du Dévonien inférieur). En revanche, les rivières du Condroz et de l'Entre-Vesdre-et-Meuse ont un régime des débits moins contrasté, dans la mesure où une partie de leur bassin hydrographique s'étend sur des terrains perméables (calcaires du Carbonifère et craies du Crétacé).

Les plaines alluviales des rivières les plus importantes, telles que la Lesse (5 et 6) ou l'Ourthe (11-14), sont principalement utilisées comme pâture. Par contre, dans les têtes de bassin (Ruisseau de Belleva (15), Ruisseau de la Mer (16), Ru des Waidages (22)), les fonds de vallées, étroits et difficiles d'accès, sont généralement occupés par des forêts alluviales et des plantations de résineux.

\section{MÉTHODOLOGIE}

\section{MARQUAGE}

Durant plusieurs saisons hydrologiques, de nombreuses campagnes de marquage des éléments constituant le fond du lit des rivières ont été menées de manière à déterminer les caractéristiques du transport de la charge de fond, et plus spécialement à identifier les débits de mise en mouvement des éléments caillouteux. Pour ce faire, des marquages à la peinture ont été réalisés in situ sur des dépôts émergés (généralement des seuils) afin de ne pas perturber l'agencement du matériel constituant le lit. Les caractéristiques du matériel $\left(D_{50}\right.$ et $\left.D_{90}\right)$ ont été estimées à l'aide d'une grille, suivant le protocole proposé par Kellerhals et Bray (1971) et appliqué avec succès notamment dans le Canal de Miribel (Poinsart, 1992). Des repérages topographiques ont été effectués afin de s'assurer qu'il y avait bien eu mise en mouvement et non enfouissement du matériel marqué.

Dans certaines rivières (Semois, Aisne, Lienne), des injections de galets contenant de faibles quantités de plomb ou équipés d'émetteur radio ont été réalisées en complément. Ces techniques présentent l'avantage d'augmenter nettement le taux de récupération puisque ces galets peuvent être récupérés même lorsqu'ils sont enfouis. II faut cependant prélever les galets afin d'y forer une cavité où seront logés soit la charge métallique, soit l'émetteur, et ensuite les réinjecter dans la rivière, sachant que leur position ne correspond pas nécessairement à la structure initiale du lit. En conséquence, avant d'exploiter tout résultat, il est indispensable d'attendre qu'une crue de faible importance se produise afin de permettre aux galets de retrouver leur agencement naturel. Par ailleurs, nous avons veillé à ce que la densité des galets équipés de pastilles de plomb ou d'émetteur radio ne soit pas augmentée de plus de $10 \%$ par rapport à la densité naturelle des galets, qui est égale à 2,6.

Le suivi de ces marquages en relation avec les événements hydrologiques nous a donc permis d'identifier les débits de mise en mouvement généralisé, c'est-à-dire les débits nécessaires pour mobiliser le $D_{50}$ de chaque rivière. Dans la suite de cet article, le débit à plein bord sera utilisé comme valeur de référence pour exprimer les débits de mise en mouvement.

\section{ANALYSE HYDROLOGIQUE}

Le débit à plein bord de chaque rivière a été identifié par des observations de terrain et sa récurrence déterminée par ajustement des crues maximales dans la distribution de Gumbel, en utilisant la série partielle des débits (Petit et Pauquet, 1997; Petit et al., 2005) (tabl. II). Dans cette approche, le débit seuil retenu pour garantir l'indépendance des débits à analyser correspond à la crue maximale annuelle la plus faible de la série d'observations. Cette méthode nous donne, en moyenne, entre 3 et 6 événements supérieurs à ce débit par an. Pour les rivières à charge caillouteuse développées sur un substrat imperméable et dont la superficie du bassin dépasse $500 \mathrm{~km}^{2}$, la récurrence du débit à plein bord oscille entre 0,9 et 1,5 an mais pour des rivières de dimension plus modeste (superficie inférieure à $250 \mathrm{~km}^{2}$ ), cette récurrence est généralement de l'ordre de 0,5 an, voire moins. Ce schéma a été vérifié par une analyse systématique des débits à plein bord effectuée sur plus d'une soixantaine de rivières de moyenne et haute Belgique (Petit et al., 2005).

La récurrence du débit de mise en mouvement a également été calculée en utilisant la série partielle des débits. De plus, en utilisant la courbe des débits classés propre à chacune des stations hydrologiques, nous avons calculé le nombre moyen de jours où le charriage se produit.

\section{DÉBIT SOLIDE}

Pour les têtes de bassin et les rivières de dimension modeste, le débit solide de la charge de fond a été estimé en installant des pièges à sédiments. II s'agissait de fosses creusées dans le lit des ruisseaux; un barrage était aménagé en aval et le fond était recouvert par une bâche ou une moustiquaire. En relation avec les événements hydrologiques, le 
TABLEAU II

Valeurs de débit de mise en mouvement (exprimé par rapport au débit à plein bord) et nombre de jours de charriage dans les rivières du massif ardennais

\begin{tabular}{|c|c|c|c|c|c|c|c|c|c|c|}
\hline & $\mathrm{N}^{\circ}$ & $\begin{array}{l}\text { Taille } \\
\left(\mathrm{km}^{2}\right)\end{array}$ & $\begin{array}{c}Q_{b}^{*} \\
\left(m^{3} / s\right)\end{array}$ & $\begin{array}{c}\text { Récurrence } \\
\mathrm{Q}_{\mathrm{b}} \text { (série } \\
\text { partielle) }\end{array}$ & $\begin{array}{l}\mathrm{Q}^{*} \text { de mise } \\
\text { en } \\
\text { mouvement } \\
\left(\mathrm{Q}_{\mathrm{mm}}\right) \\
\left(\mathrm{m}^{3} / \mathrm{s}\right)\end{array}$ & $\mathrm{Q}_{\mathrm{mm}} / \mathrm{Q}_{\mathrm{b}}$ & $\begin{array}{c}\text { Récurrence } \\
\mathrm{Q}_{\mathrm{mm}} \text { (série } \\
\text { partielle) }\end{array}$ & $\begin{array}{c}\text { Nombre de } \\
\text { jours de } \\
\text { charriage/an }\end{array}$ & $\begin{array}{c}\text { Série } \\
\text { d'observations } \\
\text { hydrologiques }\end{array}$ & Référence \\
\hline Rulles d'Anlier & 1 & 16,2 & 1,3 & 0,40 & 1,05 & 0,80 & 0,35 & 19 & 1973-1980 & Petit, 1987 \\
\hline Semois Membre & 2 & 1235 & 130 & 0,44 & 130 & 1 & 0,44 & 10,1 & 1980-2004 & Gob et al., 2005 \\
\hline Lesse Eprave & 5 & 419 & 36 & 1,9 & $<Q_{b}$ & $<1$ & & & & Petit et al., 1996 \\
\hline Lesse Villers/lesse & 6 & 1090 & 105 & 1,0 & $<Q_{b}$ & $<1$ & & & & Petit et al., 1996 \\
\hline Lhomme Eprave & 8 & 474 & 52 & 0,92 & 35 & 0,7 & 0,63 & 12 & 1969-1991 & Petit et al., 1996 \\
\hline Ourthe orientale & 10 & 192 & 21 & 1,2 & 8,4 & 0,4 & & 15 & & Petit et al., 1996 \\
\hline Ourthe Hotton & 11 & 940 & 85 & 0,61 & 81 & 0,95 & 0,55 & 5,2 & 1979-2005 & \\
\hline Ourthe Durbuy & 12 & 1215 & 100 & 0,54 & 98 & 0,98 & 0,53 & 5,3 & 1997-2005 & \\
\hline Ourthe Hamoir & 13 & 1607 & 160 & 1,10 & 120 & 0,75 & 0,52 & 6,3 & $1966-2005$ & \\
\hline Ourthe Sauheid & 14 & 2904 & 300 & 0,97 & 230 & 0,77 & 0,49 & 6,3 & $1980-2005$ & Petit et al., 1996 \\
\hline Belleva & 15 & 12,5 & 0,8 & - & 0,45 & & & 6 & $1972-1973$ & Mercenier, 1973 \\
\hline Mer & 16 & 1,4 & 0,18 & - & 0,08 & 0,45 & & 14 & $1972-1973$ & Mercenier, 1973 \\
\hline Aisne Juzaine & 18 & 183 & 23,8 & 1,51 & 10,9 & 0,46 & 0,30 & 11 & $1975-2005$ & \\
\hline Chavanne Bra & 19 & 12 & 2,9 & - & $1,3-1,7^{(\mathrm{d})}$ & $0,45-0,6^{(d)}$ & & & $2002-2005$ & \\
\hline Lienne Lorcé & 20 & 146 & 21,3 & 0,66 & 9,4 & 0,44 & 0,30 & 16 & 1977-2005 & \\
\hline Amblève Targnon & 21 & 807 & 86 & 0,53 & 62 & 0,72 & 0,41 & 10,5 & $1998-2005$ & Houbrechts, 2005 \\
\hline Waidage (1) & 22 & 0,26 & $0,04^{(b)}$ & & 0,035 & 0,88 & $0,6^{(\mathrm{e})}$ & 0,2 & 1989-1992 & Assani et Petit, 1995 \\
\hline Waidage (2) & 22 & 0,26 & $0,04^{(b)}$ & & 0,025 & 0,63 & $0,2^{(\mathrm{e})}$ & 2,0 & 1989-1992 & Assani et Petit, 1995 \\
\hline Hoëgne Solwaster & - & 19,4 & & & 3,12 & & 0,26 & 8,6 & $1993-2005$ & \\
\hline Hoëgne Pepinster & 23 & 189 & 36,8 & 1,0 & 17 & 0,46 & 0,34 & 8,3 & $1971-2005$ & \\
\hline Vesdre Pepinster & 24 & $565^{\text {(a) }}$ & 90 & $2,8^{(c)}$ & 53 & 0,59 & 0,5 & 4,9 & $1992-2005$ & \\
\hline Wavelinse & 25 & 4,3 & 0,2 & & 0,15 & 0,75 & & 11 & & Dave, 1975 \\
\hline Berwinne Dalhem & 26 & 118 & 13,8 & 1,5 & 4 & 0,29 & 0,20 & 15,5 & 1967-2005 & \\
\hline Gueule Sipenaken & 27 & 121 & 12,5 & 0,9 & 8 & 0,64 & 0,35 & 4,1 & $1994-2005$ & \\
\hline Gueule Hommerich & 28 & 144 & 14,2 & 0,9 & 10 & 0,70 & 0,45 & 3 & $1970-2005$ & \\
\hline Gueule Meersen & 29 & 329 & 24 & 2,4 & 24 & 1 & 2,4 & 0,8 & $1970-2005$ & \\
\hline
\end{tabular}

* Débit journalier. Les débits à plein bord de ces stations ont été publiés dans Petit et Pauquet (1997) et dans Petit et al. (2005).

Ru des Waidages: (1) système avec embâcles (2) système sans embâcles

(a) En aval de la confluence avec la Hoëgne.

(b) Valeur du débit dont la récurrence est de 1 an.

(c) Régime des débits influencé par des barrages écrêteurs.

(d) Mobilisation dans le secteur en step-pool pour $0,45 Q_{b}$ mais, dans le secteur à méandres, la mobilisation ne débute que pour $0,6 Q_{b}$.

(e) Inverse du nombre d'événements atteints ou dépassés par an.

piège était périodiquement vidé et les sédiments piégés étaient cubés et pesés.

Pour les rivières plus importantes, l'importance du charriage a été estimée sur la base du relevé des quantités de sédiments régulièrement retirées du lit, sachant que ces curages sont systématiquement effectués aux mêmes endroits et que ces sites jouent finalement un rôle analogue à celui d'un piège à sédiments, sachant également que des profils de contrôle sont effectués (suivant un espacement inférieur à la largeur de la rivière) avant et après chaque curage, afin de s'assurer que l'on revient aux cotes altimétriques initiales. Cette méthodologie a été développée en détail par Gob et al. (2005), notamment à la suite de travaux de curage effectués dans la Semois. Nous avons ensuite converti en masse les données exprimées en volume. Pour ce faire, nous avons utilisé une densité apparente de 1,6 qui correspond à la densité moyenne de plusieurs mesures que nous avons effec- tuées sur des échantillons prélevés dans des dépôts fluviatiles.

\section{RÉSULTATS ET DISCUSSION}

\section{FRÉQUENCE DU CHARRIAGE}

Les observations concernant le débit de début de charriage et sa fréquence sont reportées au tableau II. Dans la grande majorité des cas, il apparaît que le charriage débute pour des débits inférieurs au débit à plein bord $\left(Q_{b}\right)$. Seules se présentent deux exceptions, la Semois (2) et la Gueule dans la partie inférieure de son cours (29), sur lesquelles nous reviendrons plus en détail par la suite.

Le débit de mobilisation a globalement une récurrence de l'ordre de 0,5 an, rarement plus, souvent moins. Toutefois, malgré ces récurrences relativement faibles, le charriage ne se 
produit que quelques jours par an en moyenne: schématiquement entre 5 et 10 jours par an.

Si l'on analyse les données plus en détail, on peut dégager les points suivants. Pour des rivières ardennaises de dimension importantes (superficie supérieure à $500 \mathrm{~km}^{2}$ ), le débit de mobilisation est relativement proche du débit à plein bord: ce rapport est de 0,75 pour l'Amblève (21) et l'Ourthe inférieure (13 et 14), il est proche de 1 pour l'Ourthe moyenne (11 et 12) ; ce qui nous donne des récurrences variant de 0,4 à 0,55 an, avec seulement 5 à 6 jours de charriage par an pour l'Ourthe et 10 jours pour l'Amblève. Dans le cas de la Semois (2) qui est la seule rivière importante du sud de l'Ardenne, le débit de mobilisation est proche du débit à plein bord mais ce dernier, vu les caractéristiques morphologiques de la rivière, est atteint beaucoup plus souvent que dans les rivières du nord de l'Ardenne, de telle sorte que le nombre de jours de charriage est comparable sinon supérieur à celui observé dans les rivières du nord de l'Ardenne de taille similaire.

Dans les rivières ardennaises de taille intermédiaire, c'està-dire pour des superficies comprises entre 100 et $500 \mathrm{~km}^{2}$ (Aisne, Lienne, Hoëgne, Ourthe orientale), on constate que le charriage débute pour un débit représentant moins de 0,5 fois le débit à plein bord, avec des récurrences de l'ordre de 0,3 an et une durée de charriage variant entre 8 et 12 jours par an. II en est partiellement de même pour les rivières de l'EntreVesdre-et-Meuse: la Gueule, dans la partie supérieure de son cours (stations de Sipenaken et d'Hommerich, 27 et 28), rejoint assez bien les caractéristiques de ces rivières ardennaises, même si le charriage y débute pour un débit un peu plus élevé $\left(0,6\right.$ à $\left.0,7 Q_{b}\right)$ avec des récurrences de l'ordre de 0,4 an et surtout un nombre de jours nettement inférieur (seulement 4 jours). Malgré cela, la Gueule reste encore active, notamment en terme d'érosion latérale et de mobilité de charge (Stam, 2002; Mols, 2004), alors que dans la partie inférieure de son cours (29), on assiste à un changement radical qui se traduit par une atténuation du charriage, de la mobilité du lit et de l'érosion latérale. En revanche, dans la Berwinne (26), le charriage débute pour un débit représentant moins du tiers du débit de plein bord et se produit plus de 15 jours par an avec une récurrence de 0,2 an. Ce charriage nettement plus fréquent que pour les rivières ardennaises peut s'expliquer de la façon suivante. Dans cette partie de son cours, la Berwinne traverse les terrasses de la Meuse et l'essentiel de sa charge de fond provient du remaniement de ces terrasses, de telle sorte que le matériau qui constitue son lit est de dimension assez modeste $\left(D_{50}=40 \mathrm{~mm}\right)$. La Berwinne se retrouve en quelque sorte avec une charge sous calibrée, ce qui explique qu'elle peut la mobiliser beaucoup plus souvent (Hallot et al., 2003).

Peu d'informations sont disponibles pour les rivières de dimension plus modeste (superficie inférieure à $100 \mathrm{~km}^{2}$ ). Toutefois, la Rulles (1) montre une récurrence fort faible du débit de mobilisation (0,35 an) et un nombre élevé de jours de charriage (près de 20 jours par an) bien que les observations portent sur une période anormalement sèche (Petit, 1987). Comme nous le verrons par la suite (tabl. III), ceci est notamment à mettre en relation avec la taille du matériau constituant le lit de la rivière.
Dans les ruisseaux situés en tête de bassin tels que les ruisseaux de la Mer (16), de Belleva (15) et du Fond de Wavelinse (25), étudiés respectivement par Mercenier (1973) et par Dave (1975), la série des débits est trop courte pour calculer la récurrence du débit de charriage et celle du débit à plein bord. II apparaît également que, pour cette gamme de rivières, le charriage fonctionne peu souvent, entre 6 et 15 jours par an, selon la taille du bassin. Toutefois, il est fort probable que le nombre de jours de charriage dans ces ruisseaux soit sous-estimé puisque ces auteurs ont travaillé au cours de périodes sèches. Par ailleurs, ces bassins sont situés sous couvert forestier et, comme l'ont montré les expériences menées dans le Ru des Waidages (22), la prolifération des embâcles végétaux qui font office de barrage jouent un rôle primordial dans la réduction du charriage, suite à l'accroissement de la rugosité et à la dissipation d'énergie dans les plunge-pool (Assani et Petit, 1995).

II ressort de notre analyse que, dans les rivières ardennaises, le débit de mobilisation est nettement inférieur au débit à plein bord (entre 0,4 et $0,8 Q_{b}$ ), qu'il se présente le plus souvent avec une récurrence de l'ordre de 0,5 an et qu'il agit schématiquement entre 5 et 10 jours par an. Ceci rejoint partiellement les résultats de différentes études (tabl. I) que nous avons présentés dans l'introduction. On note toutefois certaines anomalies liées au contexte régional. II s'agit notamment de la Semois d'une part, où, vu la morphologie de son lit, la fréquence du charriage est élevée pour une rivière de cette importance et de la Berwinne d'autre part, qui présente elle aussi un charriage fréquent vu la petite dimension de sa charge de fond liée à un héritage géomorphologique (remaniement des terrasses de la Meuse).

\section{QUANTITÉS CHARRIÉES}

Les quantités charriées sont reprises au tableau III. Nous y avons également reporté les puissances spécifiques au niveau du plein bord de façon à caractériser l'énergie de ces rivières.

Pour la plus importante d'entre elles, l'Ourthe, on constate que les quantités charriées ne sont finalement pas très importantes puisqu'elles représentent seulement $0,73 \mathrm{t} / \mathrm{km}^{2} / \mathrm{an}$ dans sa partie amont (11) et de l'ordre de $0,5 \mathrm{t} / \mathrm{km}^{2}$ an dans sa partie inférieure. Dans ce dernier tronçon, on note une augmentation entre Durbuy (12) et Hamoir (13), de 0,44 à 0,54 t/km²/an qui résulte des apports de l'Aisne (2,17 t/ $\left.\mathrm{km}^{2} / \mathrm{an}\right)$. L'importance des quantités charriées par cet affluent avait déjà été mise en évidence par la comparaison des compositions pétrographiques de la charge de fond en amont et en aval de la confluence avec l'Aisne (Duchesne et Pissart, 1985). Par ailleurs, dans l'Ourthe inférieure, on peut constater que la quantité transportée sous l'effet d'une crue trentenale correspond au double de la valeur moyenne annuelle. Dans la Semois (2), il apparaît que les quantités charriées représentent plus du double de celles transportées par l'Ourthe, malgré une puissance spécifique au plein bord relativement faible. Ceci s'explique notamment par le nombre de jours de charriage plus élevé ( 10 jours/an contre 5 jours/an pour l'Ourthe) ainsi que par des formes du lit moins développées, ce qui 
TABLEAU III

Quantités charriées et puissance spécifique à plein bord

\begin{tabular}{|c|c|c|c|c|c|c|c|c|}
\hline Station & $\mathrm{N}^{\circ}$ & $\begin{array}{l}\text { Taille } \\
\left(\mathrm{km}^{2}\right)\end{array}$ & $\begin{array}{l}\text { Quantité } \\
\text { charriée } \\
\text { (t/km²/an) }\end{array}$ & $\begin{array}{l}\text { Nombre de } \\
\text { couple de } \\
\text { valeurs }\end{array}$ & $\begin{array}{l}\text { Période de } \\
\text { référence }\end{array}$ & Référence & $\begin{array}{c}\mathrm{D}_{50} \mathrm{du} \text { lit } \\
\text { (seuil) (mm) }\end{array}$ & $\begin{array}{c}\text { Puissane } \\
\text { spécifique au } \\
Q_{b}{ }^{*} \\
\left(\mathrm{~W} / \mathrm{m}^{2}\right)\end{array}$ \\
\hline Rulles d'Anlier & 1 & 16,07 & 0,02 (b) & 2 & $1977-1979$ & Petit, 1987 & 12 & 17 \\
\hline Semois (Ardenne) & 2 & $1070-1235$ & 1,12 & 4 & $1994-2001$ & Gob et al., 2005 & 70 & 23 \\
\hline Eau Noire Couvin & 3 & 180 & 1,80 & 2 & 1993-2004 & & 95 & 60 \\
\hline Lesse Han/Lesse & 4 & 387 & 1,43 & 2 & $1993-2001$ & & 48 & 50 \\
\hline Lhomme Rochefort & 7 & 429 & 1,34 & 2 & $1993-2003$ & & 58 & 83 \\
\hline Wamme Harsin & 9 & 55 & 2,80 & 3 & $1991-2004$ & & 63 & 109 \\
\hline Ourthe orientale & 10 & 192 & $3,9^{\text {(c) }}$ & 1 & & & & \\
\hline Ourthe Hotton & 11 & 940 & 0,73 & 7 & 1963-1990 & & 44 & 41 \\
\hline Ourthe Durbuy & 12 & 1285 & 0,44 & 9 & $1959-1990$ & & 44 & 54 \\
\hline Ourthe Hamoir & 13 & 1597 & 0,54 & 11 & $1959-1990$ & & 78 & 54 \\
\hline Ourthe inférieure & 14 & 2691 & $0,53\left(1,13^{(d)}\right)$ & 9 & $1951-1993$ & & 70 & 51 \\
\hline Mer & 16 & 1,36 & $0,56^{(\mathrm{b})}$ & 1 & $1972-1973$ & Mercenier,1973 & 20 & 51 \\
\hline Aisne Erezée & 17 & 66 & 2,53 & & & & 84 & 86 \\
\hline Aisne Bomal & 18 & 190 & 2,17 & & & & 92 & 92 \\
\hline Waidages & 22 & 0,26 & $0,3^{(b)}$ & 1 & 1989-1992 & Assani et Petit, 1995 & $9,7-22$ & $25^{(\mathrm{e})}$ \\
\hline Hoëgne Pepinster & 23 & 220 & 1,116 & 2 & $1981-1987$ & Deroanne, 1995 & 70 & 140 \\
\hline Vesdre Pepinster (a) & 24 & 350 & 0,704 & 1 & $1981-1987$ & Deroanne, 1995 & 70 & 79 \\
\hline Wavelinse & 25 & 4,33 & $0,36^{(b)}$ & 1 & $1973-1974$ & Dave, 1974 & 9,8 & 18 \\
\hline
\end{tabular}

* Une analyse détaillée des puissances spécifiques au plein bord de ces stations a été publiée dans Petit et al. (2005).

(a) Amont de la confluence avec la Hoëgne.

(b) Pièges à sédiments.

(c) Pour une crue décennale.

(d) Pour une crue de récurrence de 34 ans.

(e) Pour un débit de récurrence 1 an.

entraîne une rugosité moins importante et laisse donc une plus grande part d'énergie pour le charriage (Gob et al., 2005).

C'est dans des rivières de dimension moyenne (superficie comprise entre 100 et $500 \mathrm{~km}^{2}$ ) à forte énergie que les quantités charriées sont les plus importantes: plus de $2 \mathrm{t} / \mathrm{km}^{2} / \mathrm{an}$ pour la Wamme et l'Aisne (avec des puissances spécifiques de l'ordre de $100 \mathrm{~W} / \mathrm{m}^{2}$ ). Dans les autres rivières (Lesse, Lhomme, et Eau Noire), on se maintient à des valeurs voisines ou supérieures à $1,5 \mathrm{t} / \mathrm{km}^{2} / \mathrm{an}$, ce qui représente le triple des quantités charriées par l'Ourthe inférieure. On notera également que, dans l'Ourthe orientale, la quantité charriée sous l'effet d'une seule crue décennale dépasse largement $3 \mathrm{t} / \mathrm{km}^{2} / \mathrm{an}$. Dans la Hoëgne (23), rivière qui a pourtant les puissances spécifiques les plus élevées parmi les rivières étudiées $\left(140 \mathrm{~W} / \mathrm{m}^{2}\right)$, les quantités charriées restent relativement modestes (1,12 t/km²/an), malgré un $D_{50}$ relativement petit par rapport à sa puissance spécifique au plein bord. Ceci peut vraisemblablement s'expliquer par un déficit de charge de fond. En effet, la Hoëgne incise la roche en place sur 20 à $25 \%$ de la longueur totale de son linéaire (Mols, 2004). Cette incision atteint parfois $2 \mathrm{~m}$ de telle sorte que la rivière est déconnectée de sa nappe alluviale caillouteuse et qu'elle ne peut donc plus se recharger latéralement. L'origine de cette incision est toutefois difficile à expliquer, car cette rivière n'est pas en déficit de sédiments puisque la plus grande partie de la nappe caillouteuse héritée de la dernière période glaciaire n'a pas encore été déblayée. II faut toutefois rappeler que cette rivière coule dans la région du massif ardennais (les Hautes Fagnes) où se trouvent le plus de rivières en contact avec la roche en place. C'est également cette région de l'Ardenne qui s'est soulevée le plus au cours du Pleistocène moyen et supérieur (Demoulin, 1995, 1998). II n'est donc pas exclu que des processus liés à la néotectonique puissent être, au moins partiellement et peut-être avec un certain retard, à l'origine de cette incision.

Les quantités évacuées par charriage sont peu importantes dans les têtes de bassins pourtant fortement pentus. Dans le ruisseau de la Mer (16) et le ruisseau des Waidages (22), la taille de la charge de fond ne peut pas être mise en cause étant donné que le $D_{50}$ est relativement peu élevé (<22 mm) vis-à-vis de la puissance développée par ces cours d'eau (17$51 \mathrm{~W} / \mathrm{m}^{2}$ ). Mais, rappelons qu'il s'agit de bassins forestiers où les nombreux embâcles végétaux jouent un rôle primordial dans la réduction du charriage.

Par ailleurs, dans la Rulles, on note de très faibles quantités charriées malgré la fréquence élevée du débit de mobilisation. Mais dans cette partie du cours, la méandration est très prononcée (indice de sinuosité supérieur à 1,5), ce qui implique des pertes d'énergie pour vaincre les formes et laisse peu d'énergie disponible pour le charriage; ceci ressortait d'ailleurs bien du faible ratio entre les tensions de cisaillement aux grains (grain shear stress) et la tension de cisaillement totale (Petit, 1990). De plus, le rapport d'encaissement (largeur du lit majeur/lit mineur) est fort élevé (proche de 15); en 
d'autres termes, la rivière divague dans une large plaine alluviale, ce qui implique qu'elle est moins fréquemment en contact avec ses versants, là où elle pourrait se recharger en matériau. Vu cette absence de contact, la disponibilité en matériau est seulement assurée par l'érosion latérale des berges dans lesquelles se trouvent des lentilles alluviales mais cette érosion latérale est très lente, le maximum observé en trente ans se limitant à moins de $2 \mathrm{~m}$. Par ailleurs, l'indigence du charriage ressort bien puisque des cailloux marqués depuis plus de dix ans sont toujours présents dans les secteurs de marquages. Ceci serait bien entendu inconcevable dans des rivières où le charriage serait fort actif, car ces cailloux seraient enfouis dans la sous couche ou dispersés sur de longues distances.

Différentes équations ont été proposées dans la littérature pour estimer le débit solide des rivières par charriage (Gob et al., 2005). Une d'entre-elles, définie par Bagnold (1977, 1980, 1986), fait intervenir l'excès de puissance spécifique comme descripteur de l'importance du charriage. Grâce à cette équation, il est possible d'estimer la quantité de sédiments transportés par une ou plusieurs crues. Dans la même optique, nous avons analysé l'importance du charriage dans les rivières ardennaises en fonction de la puissance spécifique atteinte au plein bord. Ce débit présente l'avantage de pouvoir être utilisé comme référence pour comparer les rivières entre-elles et de correspondre plus que probablement, dans le cas des rivières ardennaises, au débit le plus efficace pour le charriage, en terme de fréquence et de travail effectué. Pour ce faire, nous avons reporté sur une même relation l'évolution des quantités charriées en fonction de la puissance spécifique au plein bord (fig. 2). Lorsque l'on prend en considération tous les points, le coefficient de détermination est médiocre $\left(R^{2}=0,40\right)$. Toutefois, si l'on écarte la Hoëgne (23) et la Semois (2), pour les raisons évoquées ci-dessus, ce coefficient s'améliore très nettement $\left(R^{2}=0,69\right)$. II se dessine un groupe de rivières à forte énergie et à grande disponibilité en sédiments, rivières de taille moyenne qui toutes descendent des reliefs marqués du nord de l'Ardenne: l'Aisne (17 et 18), le Wamme (9), la Lhomme (7) et dans une moindre mesure la Lesse (4) et l'Eau Noire (3). L'Ourthe inférieure (1214) et la Vesdre (24) montrent des quantités charriées relativement faibles vis-à-vis de leur puissance; il n'est pas exclu qu'ici aussi joue le manque de disponibilité en sédiments dans la mesure où ces deux rivières (et spécialement l'Ourthe) ont été le siège de curages répétés de longue date et soumis à des protections de berges importantes, ce qui limite l'érosion latérale et donc l'approvisionnement en sédiments.

Notons que pour des puissances spécifiques identiques, plusieurs facteurs peuvent expliquer des différences de quantités charriées. Tout d'abord, ces quantités charriées représentent des valeurs moyennes qui ne couvrent pas nécessairement les mêmes périodes d'observation pour les différentes stations. Certaines valeurs concernent des périodes sèches où l'occurrence du charriage a été limitée, d'autres des périodes où au contraire le charriage s'est présenté fréquemment. D'autre part, on peut également avoir des différences de quantités charriées d'une rivière à l'autre, à puissance égale, parce que l'effet de saillie ou, au contraire,

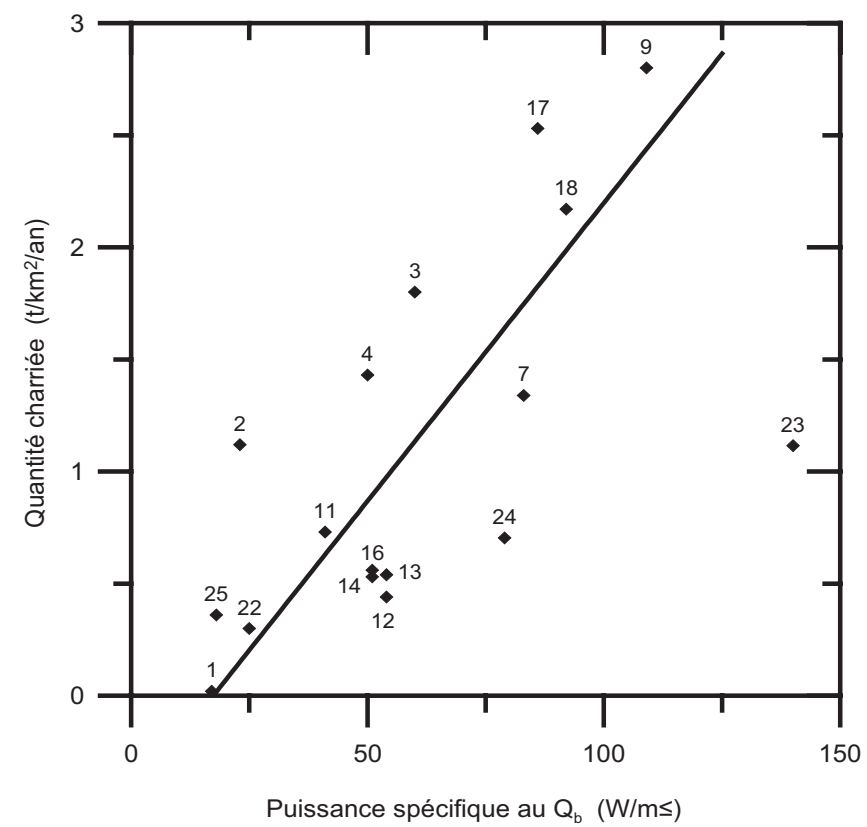

FIGURE 2. Évolution des quantités charriées en fonction de la puissance spécifique au plein bord. La droite de régression $(y=0,0266 x$ $-0,4616)$ est calculée sur l'ensemble des points à l'exception de la Semois (2) et de la Hoëgne (23). Le coefficient de détermination est de 0,69 .

Relationship between sediment yield and specific stream power at bankfull stage. The regression line $(y=0.0266 x-0.4616)$ was calculated from the whole points except those from the Semois River (2) and the Hoëgne River (23). The coefficient of determination is 0.69.

l'effet de masquage peuvent intervenir, et donc favoriser ou au contraire diminuer le charriage (Andrews, 1983; Petit, 1994).

Malgré les nuances indiquées ci-dessus, on peut néanmoins considérer que les quantités charriées par les rivières du massif ardennais sont modestes comparativement à celles quantifiées dans d'autres systèmes morphoclimatiques tels que les montagnes méditerranéennes ou des milieux semiarides, voire également dans des milieux plus comparables au nôtre (tabl. IV). Ainsi, nos valeurs se rapprochent de celles mises en évidence dans des petits bassins des Appalaches où Clément (cité par Liébault, 2003) propose des valeurs de 1,08 à $4,14 \mathrm{t} / \mathrm{km}^{2} / a n$. Différentes études réalisées au Pays de Galles et en Écosse avancent des valeurs comprises entre 2,9 et $44 \mathrm{t} / \mathrm{km}^{2} / \mathrm{an}$. Toutefois, dans ces milieux on notera une grande variabilité des résultats. Ainsi, une étude réalisée au Pays de Galles montre que les zones forestières connaissent un transport de charge de fond beaucoup plus important que les zones de prairie (Newson, 1980, 1981). De même, une étude comparative de la charge de fond réalisée en Écosse met en évidence des différences significatives entre des bassins forestiers et des bassins occupés par des landes: les productions de sédiments sont plus importantes sous forêt, notamment du fait de perturbations liées aux plantations et aux coupes, à la mise en place de drains et à la suppression de la végétation sous forêt (Stott, 1997). Ceci va à l'encontre de nos observations où les rivières de petite dimension situées sous couvert forestier transportent très peu de sédi- 
TABLEAU IV

Exemples de quantités charriées dans des rivières à charge de fond caillouteuse et à blocs

\begin{tabular}{|c|c|c|c|c|c|c|}
\hline & & Affectation du sol & $\begin{array}{l}\text { Taille } \\
\left(\mathrm{km}^{2}\right)\end{array}$ & $\begin{array}{l}\text { Quantité } \\
\text { charriée } \\
\text { (t/km²/an) }\end{array}$ & $\begin{array}{c}\text { Taille du } \\
\text { matériau du } \\
\text { lit et (charrié) } \\
(\mathrm{mm})\end{array}$ & Référence \\
\hline L'Agly & Pyrénées françaises & & 1045 & 0,96 & galets & Serrat, 1999 \\
\hline Rock Creek & Coast Range (Oregon) & & 16,2 & 3 & & Dietrich et Dunne, 1978 \\
\hline Ribera Salada River & Catalan Pre-Pyrenees & Forêt & 222 & 20 & $48(4,3)$ & Batalla et al., 2005 \\
\hline Cyff catchment & Pays de Galles & Prairie & 3,13 & 7,80 & & Newson, 1980 \\
\hline Tanllwyth & Pays de Galles & Forêt & 0,89 & 29,05 & & Newson, 1980 \\
\hline Andrews $\mathrm{O}$. & Cascade Range (Oregon) & Forêt & 0,102 & 5,88 & & Caine et Swanson, 1989 \\
\hline Niwot Ridge Mart & Colorado Rocky Mountain & Pelouse alpine & 0,08 & 0,025 & & Caine et Swanson, 1989 \\
\hline Alon Cyff & Pays de Galles & Tourbière & 0,04 & 8,0 & & Reynolds, 1986 \\
\hline North Tynes & Écosse & & 108 & 5,56 & & Petts et Thoms, 1987 \\
\hline Esconavette & Diois (France) & Forêt de montagne & 10 & $107,2^{\text {(a) }}$ & $13-51$ & Liébault, 2003 \\
\hline Barnavette & Diois (France) & Forêt de montagne & 14 & $81,6^{(a)}$ & $16-19$ & Liébault, 2003 \\
\hline Béoux & Diois (France) & Forêt de montagne & 28 & $32,0^{(a)}$ & $35-60$ & Liébault, 2003 \\
\hline \multicolumn{7}{|l|}{ Drôme (confluence } \\
\hline avec la Rhône) & Diois (France) & Forêt de montagne & 1640 & $33,6^{(a)}$ & & Piégay et al., 2004 \\
\hline 6 bassins & Centre de l'Écosse & Forêt & $0,17-1,23$ & 9,32 & $8^{(b)}$ & Stott, 1997 \\
\hline 3 bassins & Centre de l'Écosse & Lande et tourbière & $0,24-0,55$ & 2,87 & $32^{(b)}$ & Stott, 1997 \\
\hline Sagehen Creek & Californie & & 27,2 & 0,91 & $58(26)$ & Andrews, 1994 \\
\hline Eshtemoa & Désert du Néguev & $\begin{array}{l}\text { Tapis herbeux } \\
\text { discontinu }\end{array}$ & 119 & 39 & 16 & Reid et al., 1998 \\
\hline 11 bassins & Pays de Galles & $\begin{array}{l}\text { Forêt et landes } \\
\text { avec drainage }\end{array}$ & $0,25-13,0$ & $1,2-44$ & galets & Newson, 1981 \\
\hline 21 bassins & $\begin{array}{l}\text { Val d'Aoste } \\
\text { (Alpes italiennes) }\end{array}$ & $\begin{array}{l}\text { Végétation de } \\
\text { montagne }\end{array}$ & $35-299$ & 14-1926 & & Vezzoli, 2004 \\
\hline Sherbrocke & Appalaches (Canada) & Prairie & 0,75 & 1,08 & & Clément in Liébault, 2003 \\
\hline Ruisseau Jardine & Appalaches (Canada) & Forêt & 0,94 & 4,14 & & Clément in Liébault, 2003 \\
\hline
\end{tabular}

(a) Transformation des volumes $\left(\mathrm{m}^{3}\right)$ en masse (tonne) avec une densité de 1,6.

(b) $D_{50}$ dans piège à sédiments.

ments; précisons cependant que l'exploitation forestière y est peu intense alors que les bassins cités ci-dessus semblent soumis à des déboisements importants.

Par ailleurs, en accord avec nos résultats, Rickenmann (2001) montre que l'efficience des rivières à transporter une charge de fond varie de plusieurs ordres de grandeur, les écarts les plus importants s'observant dans des rivières et torrents de petite dimension, du fait d'une grande rugosité des formes. Dans les rivières à forte capacité de transport, il se pose également le problème de la disponibilité en sédiments: la capacité de transport, souvent approchée par les équations de transport solide de type Meyer-Peter et autres, donne en fait la potentialité du transport, mais cette dernière ne peut être satisfaite que si suffisamment de matériau est disponible (Kondolf et al., 2002). Ce point concorde avec les observations réalisées sur la Hoëgne et dans une moindre mesure sur l'Ourthe et la Vesdre.

\section{CONCLUSIONS}

Le débit à partir duquel débute le charriage dans des rivières du massif ardennais se produit pour des valeurs comprises entre 0,3 et $1 Q_{b}$, en fonction de la taille des cours d'eau. Ainsi, dans des rivières dont le bassin versant est infé- rieur à $100 \mathrm{~km}^{2}$, la mise en mouvement de la charge de fond se produit pour des débits compris entre 0,5 et $0,8 \mathrm{Q}_{\mathrm{b}}$. Dans les rivières plus importantes (bassin versant compris entre 100 et $500 \mathrm{~km}^{2}$ ), le charriage débute en moyenne pour un débit correspondant à $0,5 Q_{b}$ (entre 0,3 et $0,7 Q_{b}$ ). Enfin, dans les rivières dont le bassin versant est supérieur à $500 \mathrm{~km}^{2}$, cette mise en mouvement se produit pour des débits légèrement inférieurs au débit à plein bord $\left(0,7\right.$ à $\left.1 Q_{b}\right)$. Ces différences liées à la taille du bassin versant se traduisent sur la récurrence du débit de charriage et sur le nombre de jours où le charriage se produit. Ainsi, dans les rivières les plus importantes (bassin versant supérieur à $500 \mathrm{~km}^{2}$ ), la récurrence du charriage est en moyenne de 0,5 an. Dans les rivières typiquement ardennaises de taille intermédiaire (bassin versant compris entre 100 et $\left.500 \mathrm{~km}^{2}\right)$, cette récurrence est légèrement plus faible $(0,4$ an) mais on note une plus grande variabilité, spécialement lorsque l'on envisage les rivières de l'Entre-Vesdre-et-Meuse (Berwinne et Gueule). Cette récurrence semble encore plus faible $(0,3$ an) pour les bassins de dimension modeste, mais peu de données sont disponibles. Dans ces dernières, la durée du charriage peut atteindre jusqu'à 20 jours par an alors qu'elle est de deux à quatre fois moins longue dans des rivières dont le bassin versant est supérieur à 500 km². 
Les quantités charriées sont relativement peu importantes dans les bassins forestiers (de l'ordre de $0,5 \mathrm{t} / \mathrm{km}^{2} / \mathrm{an}$ ), notamment en relation avec la présence de nombreux embâcles végétaux. Pour les rivières plus importantes, où ces effets d'embâcles ne jouent qu'un rôle négligeable, les quantités charriées restent néanmoins modestes (entre 0,4 et $2,5 \mathrm{t} / \mathrm{km}^{2} / \mathrm{an}$ ), bien que la nappe de cailloutis héritée de la dernière période froide n'ait pas encore été complètement évacuée.

Ces valeurs sont relativement faibles en regard de ce qui est généralement présenté dans la littérature, mais ces dernières sont généralement obtenues dans des régions de montagnes soumises à des précipitations violentes. II est intéressant de constater que nos valeurs sont du même ordre de grandeur que les rares données portant sur des rivières situées dans des contextes géomorphologiques semblables au massif ardennais.

Enfin, la puissance spécifique au plein bord est un indicateur relativement fiable de l'importance du transport solide. La relation entre les quantités charriées et les puissances spécifiques au plein bord a permis d'identifier certaines rivières présentant un déficit en sédiments, soit à la suite de curage et de protection de berges, soit par incision dans la roche en place.

\section{REMERCIEMENTS}

Les auteurs remercient P. Ramez et un autre lecteur anonyme pour leurs commentaires apportés lors de la lecture critique du manuscrit. Un nombre appréciable de données hydrologiques, de documents topographiques et d'archives diverses ont été aimablement mis à notre disposition par la Direction des Cours d'Eau Non Navigables (DCENN), Ministère de la Région Wallonne et le Ministère de l'Équipement et des Transports, Service d'Études hydrologiques (MET) et le Service des voies navigables du District de Liège. La traduction du résumé en anglais a été corrigée par Stéphanie Mills et Natasha Shields, que nous remercions vivement.

\section{RÉFÉRENCES}

Andrews, E.D., 1983. Entrainment of gravel naturally sorted riverbed material. Geological Society of American Bulletin, 94: 1225-1231.

Andrews, E.D., 1994. Marginal bed load transport in a gravel bed stream, Sagehen Creek, California. Water Resources Research, 30: 2241-2250.

Assani, A.A. et Petit, F., 1995. Log-jams effect on bed-load mobility from experiments conducted in a small gravel-bed forest ditch. Catena, $25: 117-126$.

Bagnold, R.A., 1977. Bed load transport by natural rivers. Water Resources Research, 13: 303-312.

Bagnold, R.A., 1980. An empirical correlation of bedload transport rates in flumes and natural rivers. Proceedings of the Royal Society of London, 372A : 453-473.

Bagnold, R.A., 1986. Transport solids by natural water flow: evidence for a worldwide correlation. Proceedings of the Royal Society of London, 405A: 369-374.

Batalla, R.J., Garcia, C. et Balasch, J.C., 2005. Total sediment load in a Maditerranean mountainous catchment (the Ribera Salada River, Catalan Pre-Pyreness, NE Spain). Zeitschrift für Geomorphologie, 49: 495-514.

Bathurst, J.C., Thorne, C.R. et Hey, R.D., 1979. Secondary flow and shear stress at rivers bends. Journal of Hydraulic Division, 105: 1277-1295.
Caine, N. et Swanson, F.J., 1989. Geomorphic coupling of hillsope and channel systems in two small mountain basins. Zeitschrift für Geomorphologie, 33 : 189-203.

Dave, C., 1975. Étude de la dynamique fluviale d'un petit ruisseau de type torrentiel. Mémoire de licence, Université de Liège, 148 p.

Demoulin, A., 1995. L'Ardenne bouge toujours: néotectonique du Massif ardennais, p. 110-135. In A. Demoulin, édit., L'Ardenne: essai de géographie physique. Livre en hommage au Professeur A. Pissart, 238 p.

Demoulin, A., 1998. Testing the tectonic significance of some parameters of longitudinal river profiles: the case of the Ardenne (Belgium, NW Europe). Geomorphology, 24 : 189-208.

Deroanne, C., 1995. Dynamique fluviale de la Hoëgne. Evaluation longitudinale des caractéristiques sédimentologiques du lit et des paramètres de mobilisation de la charge de fond. Mémoire de licence, Université de Liège, $155 \mathrm{p}$.

Dietrich, W.E. et Dunne, T., 1978. Sediment budget for a small catchment in mountainous terrain. Zeitschrift für Geomorphologie, Suppl. Bd. 29: 191206.

Duchesne, F. et Pissart, A., 1985. Valeur statistique des comptages de cailloux de différentes lithologies. Application aux alluvions actuelles de l'Ourthe. Bulletin de la Société Géographique de Liège, 21 : 13-23.

Ergenzinger, P., Schmidt, K. et Busskamp, R., 1989. The pebble transmitter system (PETS) : first results of a technique for studying coarse material erosion, transport and deposition. Zeitschrift für Geomorphologie, 33: 503-508.

Ferguson, R.I., 1994. Critical discharge for entrainment of poorly sorted gravel. Earth Surface Processes and Landforms, 19: 179-186.

Ferguson, R.I., Bloomer, D.J., Hoey, T.B. et Werritty, A., 2002. Mobility of river tracer pebbles over different timescales. Water Resources Research, 38: 1-9.

Gintz, D., Hassan, M.A. et Schmidt, K.H., 1996. Frequency and magnitude of bedload transport in a mountain river. Earth Surface Processes and Landforms, $21: 433-455$.

Gob, F., Houbrechts, G., Hiver, J.M. et Petit, F., 2005. River dredging, channel dynamics and bedload transport in an incised meandering river (the River Semois, Belgium). River Research and Applications, 21 : 791-804.

Gomez, B., Naff, R.L. et Hubbell, D.W., 1989. Temporal variations in bedload transport rates associated with the migration of bedforms. Earth Surface Processes and Landforms, 14: 135-156.

Hassan, M.A., Church, M. et Ashworth, P.J., 1992. Virtual rate and mean distance of travel of individual clasts in gravel-bed channels. Earth Surface Processes and Landforms, 17:617-627.

Hassan, M.A. et Ergenzinger, P., 2003. Use of tracers in fluvial geomorphology, p. 397-423. In G.M. Kondolf et H. Piégay, édit., Tools in fluvial geomorphology. John Wiley and Sons, Chichester, 688 p.

Hallot, E., Petit, F., Verniers, G. et Lambot, F., 2003. Utilisation des techniques végétales pour la stabilisation des berges: suivi de chantiers réalisés dans différents types de rivières wallonnes. In Actes du colloque Évaluer le milieu, Journées des géographes belges, SOBEG-BEVAS, Liège, p. 173-178.

Hoey, T.B., 1992. Temporal variations in bed load transport rates and sediment storage in gravel bed rivers. Progress in Physical Geography, 16: 319-338.

Houbrechts, G., 2005. Utilisation des macroscories et des microscories en dynamique fluviale: application aux rivières du massif ardennais (Belgique), Thèse de doctorat, Université de Liège, $328 \mathrm{p}$.

Juvigné, E., 1979. L'encaissement des rivières ardennaises depuis le début de la dernière glaciation. Zeitschrift für Geomorphologie, $23:$ 291-300.

Kellerhals, R. et Bray, D.I., 1971. Sampling procedures for coarse fluvial sediments. Journal of Hydraulic Division, 97: 1165-1180.

Kondolf, G.M., Piégay, H. et Landon, N., 2002. Channel response to increased and decreased bedload supply from land use change: contrast between two catchments. Geomorphology, 45 : 35-51.

Lamarre, H., McVicar, B. et Roy, A.G., 2005. Using passive integrated transponder (PIT) tags to investigate sediment transport in gravel-bed rivers. Journal of Sedimentary Research, 75: 736-741. 
Leopold, L.B., Wolman, M.G. et Miller, J.D., 1964. Fluvial Processes in Geomorphology. Freeman, San Francisco, 522 p.

Leopold, L.B., 1994. A View of the River. Harvard University Press, Cambridge, $298 \mathrm{p}$.

Liébault, F., 2003. Les rivières torrentielles des montagnes drômoises: évolution contemporaine et fonctionnement géomorphologique actuel (massifs du Diois et des Baronnies). Thèse de doctorat, Université Lumière Lyon II, $357 \mathrm{p}$.

Martin, Y., 2003. Evaluation of bed load transport formulae using field evidence from the Vedder River, British Columbia. Geomorphology, 53: 75-85.

Miyamoto, K., Kurihara, J., Sawada, T. et Itakura, Y., 1992. A study of field methods for measuring sediment discharge, p. 107-114. In J. Bogen, D.E. Walling, T. Day, édit., Erosion and Sediment Transport Monitoring Programmes in River Basins. International Association of Hydrological Sciences, Wallingford, IAHS Publication 210, 538 p.

Mercenier, J., 1973. Dynamique fluviale dans un petit bassin du rebord méridional du Plateau des Tailles. Mémoire de licence, Université de Liège, $148 \mathrm{p}$.

Mols, J., 2004. Dynamique fluviale en réponse aux changements d'affectation du sol des bassins versants de l'Euregio Meuse-Rhin, Mémoire de diplôme d'études approfondies, Université de Liège, 55 p.

Newson, M., 1980. The erosion of drainage ditches and its effect on bed-load yields in mid-Wales: reconnaissance case studies. Earth Surface Processes and Landforms, $5: 275-290$.

Newson, M., 1981. Mountain streams, p. 58-89. In J. Lewin, édit., British Rivers. Allen and Unwin, London, $216 \mathrm{p}$.

Petit, F., 1987. The relationship between shear stress and the shaping of the bed of a pebble-load river (la Rulles-Ardenne). Catena, 14: 453-468.

Petit, F., 1990. Evaluation of grain shear stress required to initiate movement of particles in natural rivers. Earth Surface Processes and Landforms, 15: 135-148.

Petit, F., 1994. Dimensionless critical shear stress evaluation from flume experiments using different gravel beds. Earth Surface Processes and Landforms, 19: 565-576.

Petit, F., 1995. Régime hydrologique et dynamique fluviale des rivières ardennaises, p. 194-223. In A. Demoulin, édit., L'Ardenne: essai de géographie physique. Livre en hommage au Professeur A. Pissart, 238 p.

Petit, F., Poinsart, D. et Bravard, J.P., 1996. Channel incision, gravel mining and bed-load transport in the Rhone river upstream to Lyon, France (Canal Miribel). Catena, 26:209-226.

Petit, F., Pauquet, A. et Pissart, A., 1996. Fréquence et importance du charriage dans des rivières à charge de fond caillouteuse. Géomorphologie, Relief, Processus et Environnement, 2: 3-12.

Petit, F. et Pauquet, A., 1997. Bankfull discharge recurrence interval in gravel bed rivers. Earth Surface Processes and Landforms, 22: 685-693.

Petit, F., Gob, F., Houbrechts, G. et Assani, A.A., 2005. Critical unit stream power in gravel-bed rivers. Geomorphology, 69: 92-101.

Petit, F., Hallot, E., Houbrechts, G. et Mols, J., 2005. Evaluation des puissances spécifiques de rivières de moyenne et de haute Belgique. Bulletin de la Société Géographique de Liège, 46 : 37-50.

Petts, G.E. et Thoms, M.C., 1987. Morphology and sedimentology of a tributary confluence bar in a regulated river: north Tyne, UK. Earth Surface Processes and Landforms, 12: 433-440.
Piégay, H., Walling, D.E., Landon, N., He, Q., Liébault, F. et Petiot, R., 2004 Contemporary changes in sediment yield in in an alpine mountain basin due to afforestation (the upper Drôme in France). Catena, 55: 183-212.

Poinsart, D., 1992. Effets des aménagements fluviaux sur les débits liquides et solides. L'exemple du Rhône dans les plaines de Miribel-Jonage et de Donzère-Mondragon. Thèse de doctorat, Université Jean Moulin Lyon III, $501 \mathrm{p}$.

Powell, D.M. et Ashworth, P.J., 1995. Spatial pattern of flow competence and bed load transport in a divided gravel bed river. Water Resources Research, $31: 741-752$

Reid, I. et Frostick L., 1984. Particle interraction and its effect on the thresolds of initial and final bedload motion in coarse alluvial channels. In E.H. Koster et R.J. Steel, édit., Sedimentology of gravels and conglomerates. Canadian Society of Petroleum Geologists, Memoir 10, p. 61-68.

Reid, I., Frostick, L.E. et Layman, J.T., 1985. The incidence and nature of bedload transport during flood flows in coarse grained alluvial channels. Earth Surface Processes and Landforms, 10: 33-44.

Reid, I., Laronne, J.B. et Powell, D.M., 1998. Flash-flood and bedload dynamics of desert gravel-bed streams. Hydrological Processes, 12 : 543-557.

Reynolds, B., 1986. A comparison of elements outputs in solution, suspended sediments and bedload for a small upland catchment. Earth Surface Processes and Landforms, 11:217-221.

Richards, K., 1988. Fluvial geomorphology. Progress in Physical Geography, $12: 435-456$.

Richards, K., 1990. Fluvial geomorphology: initial motion of bed material in gravel-bed rivers. Progress in physical Geography, 14 : 395-415.

Rickenmann, D., 2001. Comparison of bed load transport in torrents and gravel beds streams. Water Resources Research, 37: 3295-3305.

Serrat, P., 1999. Dynamique sédimentaire actuelle d'un système fluvial méditerranéen: I'Agly (France). Sciences de la terre et des planètes, 329: 189196.

Stam, M.H., 2002. Effect of land-use and precipitation changes on floodplain sedimentation in the nineteenth and twentieth centuries (Geul River, The Netherlands), Special Publications of the International Association of Sedimentologists, $32:$ 251-267.

Stott, T., 1997. Forestry effects on bedload yields in mountain streams. Applied Geography, 17: 55-78.

Thorne, C.R. et Lewin, J., 1979. Bank processes, bed material movement and planform development in a meandering river, p. 117-155. In D.D. Rhodes et G.P. Williams, édit., Adjustments of the fluvial system. Kendall/Hunt, Dubuque.

Tricart, J., 1965. Observations sur le charriage des matériaux grossiers par les cours d'eau. Revue de Géomorphologie Dynamique, 12: 3-15.

Tricart, J., 1977. Précis de géomorphologie: géomorphologie dynamique générale (tome 2). SEDES, Paris, $345 \mathrm{p}$.

Tricart, J. et Vogt, H., 1967. Quelques aspects du transport des alluvions grossières et du façonnement des lits fluviaux. Geografiska Annaler, 49A: 351366.

Vezzoli, G., 2004. Erosion in Western Alps (Dora Baltea Basin) : 2. Quantifying sediment yield. Sedimentary Geology, 171: 247-259. 
